\title{
Edge Detection Based on Canny-Oscillation Algorithm
}

\author{
Jun Jin \\ The Institute of Mathematics and Statistics \\ East China Normal University \\ Shanghai, China \\ 10153700108@ecnu.cn
}

\author{
Luyin $\mathrm{Fu}$ \\ The Institute of Financial Engineering \\ East China Normal University \\ Shanghai, China \\ 10143700124@ecnu.cn
}

\begin{abstract}
Based on Canny, a typical edge detection method, a generalized Canny-Oscillation algorithm of edge detection method is proposed. However, the traditional Canny algorithm bears a defect in the edge-detection of details and it is futile when noise signals are involved. To solve these problems, the group merged the oscillation theory into non-maximum suppression process in an attempt to equip the display-pixel matrices with enhanced accuracy. As can be seen in the test results, our method proves to be satisfactory and delivers better performance than traditional approaches. This strategy is still efficacious when applied to other edge-detection algorithms such as Sobel.
\end{abstract}

Keywords-Canny Algorithm, Edge Detection, Image Processing, Oscillation Theory

\section{INTRODUCTION}

As an important part in digital image processing, edge detection often requires careful handling. Traditional edgedetection operators, for instance, Roberts, Sobel, Prewitt, Lapacian, have enjoyed a huge group of users from the industrial sector. These operators are theoretically workable but often not entirely satisfactory in practical use due to their sensitiveness to noise. And the condition of an image is usually affected by various factors during the shooting process. Hence Canny algorithm by itself can hardly settle the threshold that reflects all the grey level information. In this paper, we put forward a new method in which an innovative four-direction Canny algorithm is used for prior operation and an approximation algorithm derived from oscillation theory is then employed. Our experimental results show accuracy better than that of traditional non-maximum suppression.

\section{TRADITIONAL CANNY AlgORITHM}

\section{A. Image Smoothing}

The first derivative of the two-dimensional Gauss function is used in Canny algorithm to smooth the image. We set the two- dimensional Gauss function as:

$$
\mathrm{G}(h, v)=\frac{1}{2 \pi \sigma^{2}} \exp \left(-\frac{h^{2}+v^{2}}{2 \sigma^{2}}\right)
$$

Where $\mathrm{h}$ is the horizontal vector and $\mathrm{v}$ represents the vertical component. Now we calculate the gradient vector and decompose it into two one-dimensional columns, which will be used as convolutional filters:

$$
\left\{\begin{array}{c}
\nabla G=\left[\begin{array}{l}
\partial G / \partial h \\
\partial G / \partial v
\end{array}\right] \\
\frac{\partial G}{\partial h}=c x \exp \left(-\frac{h^{2}}{2 \sigma^{2}}\right) \exp \left(-\frac{v^{2}}{2 \sigma^{2}}\right)=g_{1}(h) g_{2}(v) \\
\frac{\partial G}{\partial v}=c v e x p\left(-\frac{h^{2}}{2 \sigma^{2}}\right) \exp \left(-\frac{v^{2}}{2 \sigma^{2}}\right)=g_{1}(v) g_{2}(h)
\end{array}\right.
$$

Where $\mathrm{c}$ is a constant and $\sigma$ is the control parameter of the filters.

\section{B. Typical Gradients and Direction}

The Canny operator is concise and efficient. Let I $x$, y be an array of smoothed image data. After obtaining the grey-level image, we define the matrix operator as [6]:

$$
\left\{\begin{array}{l}
S_{h}=\left[\begin{array}{cc}
-1 & 1 \\
-1 & 1
\end{array}\right] \\
S_{v}=\left[\begin{array}{cc}
1 & 1 \\
-1 & -1
\end{array}\right]
\end{array}\right.
$$

Substituting (3) to the pixel matrix, we have:

$$
\left\{\begin{array}{l}
P_{h}[i, j]=\frac{1}{2}(I[i+1, j]-I[i, j]+I[i+1, j+1]-I[i, j+1]) \\
P_{v}[i, j]=\frac{1}{2}(I[i, j+1]-I[i, j]+I[i+1, j+1]-I[i+1, j])
\end{array}\right.
$$

Then, the amplitude and direction could be written as:

$$
\begin{gathered}
\mathrm{M}[\mathrm{i}, \mathrm{j}]=\sqrt{{P_{h}}^{2}+P_{v}{ }^{2}} \\
\mu[\mathrm{i}, \mathrm{j}]=\arctan \left(\frac{P_{v}}{P_{h}}\right)
\end{gathered}
$$

\section{Typical Non-maximum Suppression}

1) Area selection: A $3 * 3$ patch is utilized in the Canny algorithm, with the amplitude matrices of 8 different directions used in interpolation. As shown in the graph below (figure 1), we first choose a square patch around a certain point and mark out the gradient direction line of the point. dTmp1 and dTmp2 are the two points where the gradient direction line and the boundary of the selected square patch 
intersect. Their amplitudes can be calculated using the amplitude data of points from g1 to g4. For each point of the patch, we would later compare the amplitude of central point, $\mathrm{M}[\mathrm{i}, \mathrm{j}]$ with that of dTmp1 and dTmp2. The suppression operation should be executed on the area formed by the central point, the gradient line and the boundary of the $3 * 3$ patch. We donate this selected area as $\delta[\mathrm{i}, \mathrm{j}]$.

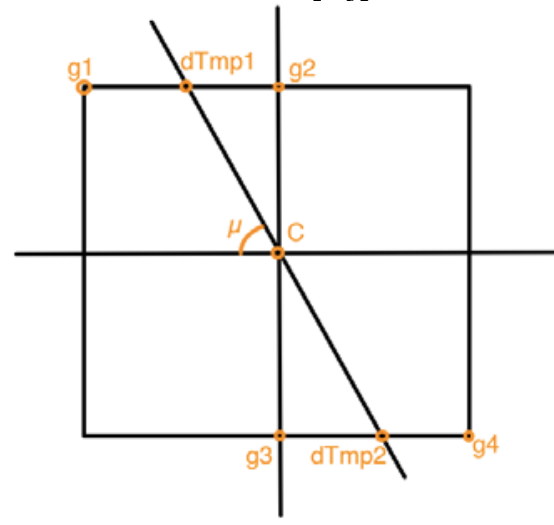

Fig. 1. Typical Non-maximum Suppression

2) Mathematical operation: If $\mathrm{M}[\mathrm{i}, \mathrm{j}]$ is smaller than the two interpolated values (the amplitude of dTmp1 and dTmp2); this particular point is considered not to be part of the edge by assigning the corresponding edge marker to 0. Eventually, this process refines the wide ridge of $\mathrm{M}$ into a pixel band without changing the gradient amplitude values in $\mathrm{M}$, which can be written as[4]:

$$
\mathrm{N}[\mathrm{i}, \mathrm{j}]=\operatorname{NMS}(M[i, j], \delta[i, j])
$$

In which "NMS" is non-maximum suppression.

\section{Dual Thresholds Method}

After calculating the amplitude matrix $\mathrm{M}$, we construct an amplitude histogram. Here we choose the upper p quantile (usually $\mathrm{p}=0.3$ ) and half of that value [9] to be respectively the upper and lower threshold. For a 3

of the point exceeds the upper threshold and one of the other eight points has amplitude less than the lower threshold, a specific edge can be delineated, and the process goes on to produce a closed curve [8].

\section{ADVANCED CANNY-OSCILLATION ALGORITHM}

\section{A. Modified Gradients and Direction}

Let $\mathrm{I}[\mathrm{x}, \mathrm{y}]$ be an array containing data of a smoothed image. After catching a patch of 3

gradients (h axis, $v$ axis, $45^{\circ}$ axis, $135^{\circ}$ axis) of the central point as[5]:

$$
\begin{aligned}
& P_{h}[i, j]=\frac{1}{2}(I[i+1, j]-I[i-1, j]) \\
& P_{v}[i, j]=\frac{1}{2}(I[i, j+1]-I[i, j-1])
\end{aligned}
$$

$$
\begin{aligned}
& P_{45^{\circ}}[i, j]=\frac{1}{2}(I[i-1, j+1]-I[i+1, j-1]) \\
& P_{135^{\circ}}[i, j]=\frac{1}{2}(I[i+1, j+1]-I[i-1, j-1])
\end{aligned}
$$

The calculation of the gradients' amplitude and direction is really a demanding job. Any operations taken should always obey vector arithmetic rules. The sample graph (figure 2) given below manifests a possible gradient direction.

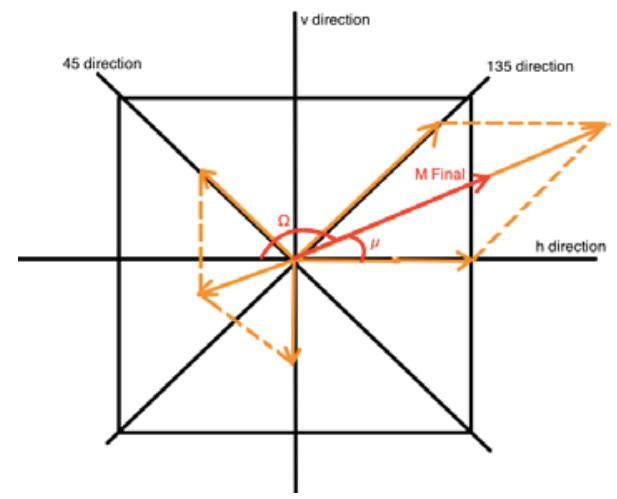

Fig. 2. Four-direction Gradient

Let $\vec{h}, \vec{v}, \vec{l}$, $\vec{j}$ be the unit vector in each direction, we obtain the resultant using [3]:

$$
\vec{M}[i, j]=P_{h} \vec{h}+P_{v} \vec{v}+P_{45^{\circ}} \vec{i}+P_{135^{\circ}} \vec{j}
$$

Therefore, we can write the amplitude as:

$$
\sqrt{\vec{M}[i, j]=}=
$$

Further, the directional angle is given by:

$$
\text { * } 3 \text { patch, i尺 } \Theta_{\text {re }} \text { arfitait }\left(\begin{array}{l}
P_{v}+\frac{\sqrt{2}}{2} P_{135^{\circ}}+\frac{\sqrt{2}}{2} P_{45^{\circ}} \\
P_{h}+\frac{\sqrt{2}}{2} P_{135^{\circ}}-\frac{\sqrt{2}}{2} P_{45^{\circ}}
\end{array}\right)
$$

\section{B. Oscillation Theory}

One part of our new theory is that we recognize the similarity between the pixel and the vibrating particle. Actually all the vibration information can be recorded in a dyadic array like (M[i,j], $\Omega[i, j])$. When a known point is recognized as source of the vibration, we simply consider its individual vibration function to be. * 3 data $m$ atrix, w e can define four

$$
\mathrm{M}(h, v, t)=A(h, v) \cos (w(h, v) t+\varphi)
$$

But to be more rigorous, given the initial condition, each $(\mathrm{h}, \mathrm{v})$ point of the plane should be obtained solving the vibration partial differential equation below of which the pixel arrangement obeys:

$$
\frac{\partial^{2} M}{\partial t^{2}}=-a \frac{\partial}{\partial x}\left(\frac{\partial M}{\partial t}\right)=-a \frac{\partial}{\partial x}\left(-a \frac{\partial M}{\partial x}\right)=a^{2} \frac{\partial^{2} M}{\partial x^{2}}
$$


The solving process has been omitted. The solution of the equation with the $M(x, 0)=\varphi(x)$ and $M t(x, 0)=\Psi(x)$ being the initial condition is called " $\mathrm{D}$ 'Alembert solution":

$$
\begin{array}{r}
\mathrm{u}(x, t)=\frac{1}{2}[\varphi(x+a t)+\varphi(x-a t)] \\
+\frac{1}{2 a} \int_{x-a t}^{x+a t} \psi(\xi) d \xi
\end{array}
$$

Moreover, the variable $\mathrm{t}$ can be obtained if the palstance is posited to be a constant. When the gradient direction angels of two pixels i.e. the phase of the vibration is known, the wave interval can be calculated using the expression:

$$
\mathrm{t}=\frac{\Omega_{2}-\Omega_{1}}{\omega}
$$

\section{Approximation Algorithm and Error Analysis}

According to vibration theory, if there exist two given points with full information (meaning both amplitude and phase data are known) and the distance between these two points is relatively short, an approximation algorithm could be conducted as:

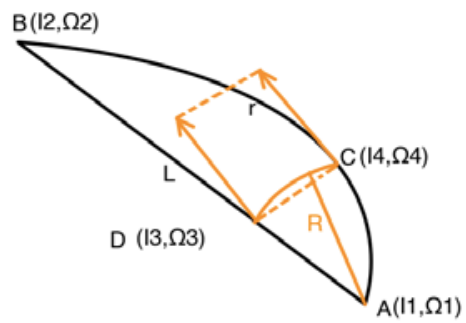

Fig. 3. Approximation Demonstration

In the figure, A and B are two points with full information and the curve is the authentic gradient path, while the straight line is the approximate path of the gradient. According to oscillation theory, the gradient state of point $\mathrm{D}$ can be easily simulated by simple linear interpolation of amplitude and phase; however, point $\mathrm{C}$ can hardly be described because of an uncharted route. A quite straightforward thought would be: what if we replace the vibration state of point $C$ with the vibration state of point $D$.

Calculation below confirms the validity of our approximation with a satisfying result.

$$
\begin{gathered}
\left\{\begin{array}{c}
t_{D}=\frac{\Omega_{2}-\Omega_{1}}{\omega} \frac{R}{L} \\
t_{C}=\frac{\Omega_{2}-\Omega_{1}}{\omega} \frac{R}{r} \\
\Delta t=t_{D}-t_{C} \\
\Delta I=I_{D}-I_{C}
\end{array}\right. \\
\approx \frac{1}{2} \dot{\varphi}(x+a t) \Delta t+\frac{1}{2} \dot{\varphi}(x-a t) \Delta t+\frac{1}{2 a} \int_{x-a \Delta t}^{x+a \Delta t} \psi(\xi) d \xi \\
\frac{\Delta I}{\Delta t} \leq \max |\dot{\varphi}|+\max |\psi|(19)
\end{gathered}
$$

Obviously, max $\left|\varphi^{\prime}\right|+\max |\Psi|$ must be lower than gradient after decomposing the trend. In other words, $\varphi$ could be the substitution of the gradient matrix $\mathbf{M}$, so the error is equivalent and commensurate to the gradient of the gradient matrix $\mathrm{M}$. Manifestly, this approach is a success with an error close to 0 .

As for the direction angle, we have:

$$
\begin{aligned}
\Delta \Omega=\Omega_{4}-\Omega_{3}= & \left.\frac{R}{r} \Omega_{2}+\frac{r-R}{r} \Omega_{1}\right) \\
& -\left(\frac{R}{L} \Omega_{2}+\frac{L-R}{L} \Omega_{1}\right)
\end{aligned}
$$

(20) can also be written as:

$$
\Delta \Omega=\Delta \mathrm{t} \omega \frac{\Omega_{1}}{\Omega_{2}-\Omega_{1}}+\left(2-\frac{\left(t_{D}+t_{C}\right) \omega}{\Omega_{2}-\Omega_{1}}\right) \Omega_{2}
$$

Where $\omega$ is a finite constant. And obviously, when $\Delta \mathrm{t} \rightarrow 0$ and $\frac{\left(t_{D}+t_{C}\right) \omega}{\Omega_{2}-\Omega_{1}} \rightarrow 2$, we have $\Delta \Omega \rightarrow 0$.

\section{Oscillation Theory in Modified Non-maximum Suppression}

One hypothesis of the typical non-maximum suppression is that the transformation of the gradient matrix $M[i, j]$ is a linear polynomial. In fact, it indeed isn't. Therefore, the application of oscillation theory in non-maximum suppression is able to produce more accurate calculation results.

While prior operation is similar to typical non-maximum suppression; a new method is used in the calculation of the node value.

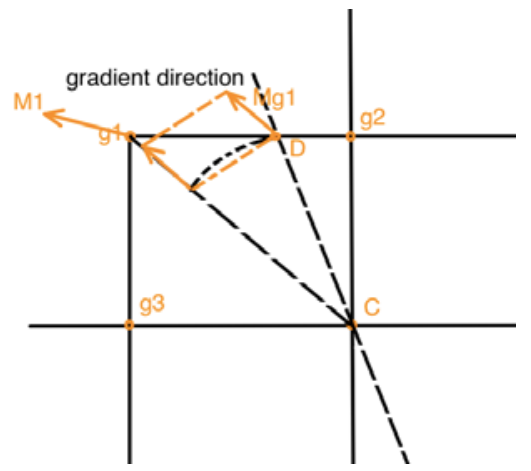

Fig. 4. Calculation of the Node

In our approach, the gradient of point $\mathrm{D}$ is influenced by that of point g1 to g4. Therefore, when the point $\mathrm{C}$ and g1 are fixed, a vectorial component Mg1 could be calculated using the approximation algorithm derived from D'Alembert solution. Let $\overrightarrow{M_{1}}$ be the gradient of the point g1 and $\overrightarrow{M_{0}}$ be the gradient of the point $\mathrm{C}$, we have:

$$
|\overrightarrow{M g 1}|=\left|\overrightarrow{M_{1}}\right| \frac{|\mathrm{CD}|}{|\mathrm{Cg} 1|}+\left|\overrightarrow{M_{0}}\right| \frac{|\mathrm{Cg} 1|-|\mathrm{CD}|}{|\mathrm{Cg} 1|}
$$

Let $\Omega_{1}$ be the gradient angle of point g1 and $\Omega_{0}$ be that of point C, we obtain:

$$
\theta_{M g 1}=\Omega_{1} \frac{|\mathrm{CD}|}{|\mathrm{Cg} 1|}+\Omega_{0} \frac{|\mathrm{Cg} 1|-|\mathrm{CD}|}{|\mathrm{Cg} 1|}
$$

It works in similar way for the other two couples of points (C \& g2, C \& g3). In this way, the resultant gradient level of D can be written as[7]: 


$$
\overrightarrow{M_{D}}=\frac{1}{3} \overrightarrow{M g 1}+\frac{1}{3} \overrightarrow{M g 2}+\frac{1}{3} \overrightarrow{M g 3}
$$

\section{EXPERIMENTAL SIMULATION AND ANALYSIS}

We have performed experiments with the CannyOscillation algorithms on images shown in figure 5(a) and figure 5(b) below. Figure 5(a) is an artificial image and figure 5(b) is the standard testing image "Lena". To investigate the algorithm's performance on anti-noise ability, both test images have been added noise process following $\mathrm{N}\left(0,10^{2}\right)$. (A rounding process is conducted when add the noise signal into the original image.)

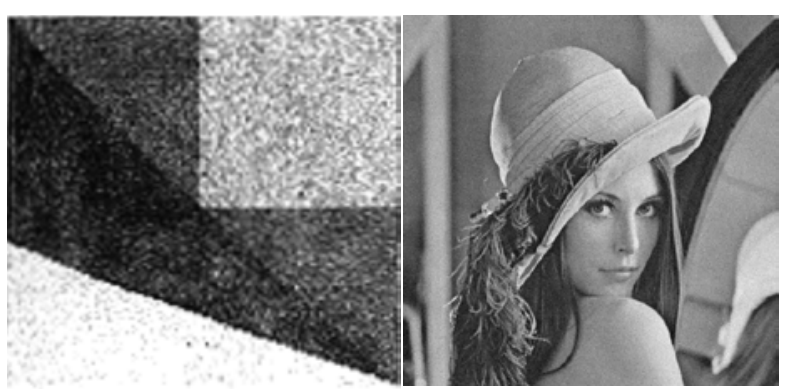

Fig. 5. (L) Artificial Image (R) Lena Image

Note that the artificial image is constituted by: top right corner with 160 as gray level, adjacent region with 128 as gray level, intermediary area with gray level 66, and the lower left quarter with grey level 210.

\section{A. Edge Detection of Figure 5(a)}

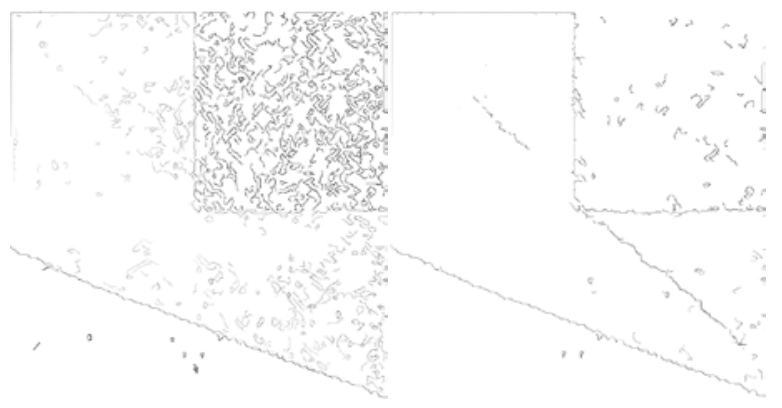

Fig. 6. (L) Canny Algorithm $\quad$ (R) Canny-Oscillation Algorithm

Figure 6(a) is created by traditional Canny algorithm while figure 6(b) is delineated by our Canny-Oscillation algorithm. Compared with figure 6(a), figure 6(b) is noticeably less affected by the noise we have added. [1]

Apart from that, the detail handling of figure 6(b) is conspicuously better than figure 6(a), hence our algorithm proves to be an improved one in this respect.

\section{B. Edge Detection of Figure 5(b)}

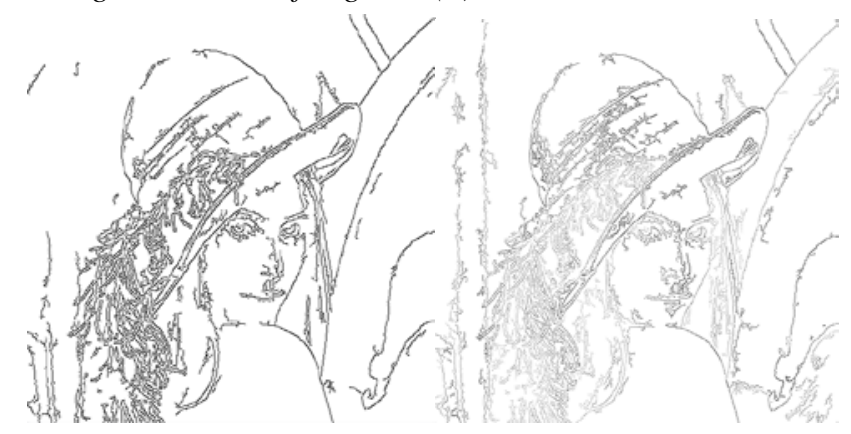

Fig. 7. (L) Canny Algorithm

(R) Canny-Oscillation Algorithm

Likewise, the edge image figure 7(b) contains more details such as the upright column and the right decoration. It is noteworthy that the edge in figure 7(b) and figure 6(b) is slenderer than that in figure 7(a) and figure 6(a). That's because the width of the pixel band declines out of high precision [2]. In other words, the detected edge using our method has a higher signal-to-noise ratio and exhibits better connectivity.

\section{CONCLUSION}

The Canny-Oscillation algorithm is an advanced variant of the traditional Canny algorithm. It maintains the advantages of the conventional Canny operator and improves the ability to suppress noise effect. Moreover, the new algorithm enjoys better detail detection ability, and the edge it obtained has a higher signal-to-noise ratio and better connectivity. What is not mentioned in the text is that the Canny-Oscillation algorithm also shows performance better than other simple operators such as Laplacian and Sobel. However, the problem of slower computing remains to be solved in further research.

\section{ACKNOWLEDGMENT}

This work was supported by the department of statistics of East China Normal University. The authors would like to thank the professor Yincai Tang and schoolmate Yiming Liu, for they have given a great deal of meritorious inspiration.

\section{REFERENCES}

[1] Tinku Acharya and Ping Sing Tsai.Edge-detection based noise removal algorithm, 2001.

[2] L.LiuandJCho.Theeffectofpixelnonlinearityoncathode-raytubebasedvisual acuity tests. Optometry \& Vision Science Official Publication of the American Academy of Optometry, 79(11):724, 2002.

[3] P. Meer and B. Georgescu. Edge detection with embedded confidence. IEEE Transactions on Pattern Analysis \& Machine Intelligence, 23(12):1351-1365, 2001.

[4] Alexander Neubeck and Luc Van Gool. Efficient non-maximum suppression. In International Conference on Pattern Recognition, pages 850-855, 2006.

[5] ZhiWangandH.E.Sai-Xian.Anadaptiveedg detection method based on canny algorithm. Journal of Image \& Graphics, 9(8):957-962, 2004.

[6] Wei Bo Wei. Study on edge detection method. Computer Engineering \& Applications, 2006. 
[7] Jian Yang and Jing Yu Yang. From image vector to matrix: a straightforward image projection techniqueâA Ťimpca vs. pca. Pattern Recognition, 35(9):1997-1999, 2002.

[8] L.I.Yu.Auto-adaptive double threshold canny operator for image edge detection. Journal of Changchun Institute of Technology, 2007.
[9] Xuan Zhu, Guo Hua Geng, Ming Quan Zhou, Yu Qing Hou, Li Yan, and Jin Ye Peng. Aapplication of two thresholds for wavelet image edge. Microcomputer Development, 2005. 